\title{
VISTAnet: Radiation therapy treatment planning through rapid dose calculation and interactive 3D volume visualization
}

\author{
Andrei State ${ }^{*}$, Julian Rosenman, MD $\$$ Henry Fuchs, ${ }^{*}$ Tim J. Cullip $\$$ and Jim Symon \\ Departments of ${ }^{*}$ Computer Science and $\$$ Radiation Oncology \\ University of North Carolina at Chapel Hill \\ Chapel Hill, North Carolina, 27514
}

\begin{abstract}
VISTAnet, an experimental gigabit network test bed, ties together a CRAY Y-MPTM, the Pixel-Planes 5 graphics engine, and an SGITM host machine to create a metacomputer capable of real-time radiation therapy dose calculation and display. We report on the methods used to manipulate and examine the $3 \mathrm{D}$ radiation dose distribution, with emphasis on the visualization, which uses a parallel, interactive, multimodal renderer implemented on Pixel-Planes 5. The real-time display is designed to facilitate comprehension of spatial relationships among the geometrically complex anatomy and radiation dose structures that characterize a $3 \mathrm{D}$ radiation treatment scenario. The currently ongoing clinical evaluation of VISTAnet has already yielded encouraging results.
\end{abstract}

\section{INTRODUCTION}

\subsection{D radiation treatment planning}

One of the major goals of the effort in 3D radiation treatment planning (RTP) is to construct treatment plans which deliver a high radiation dose to the planning target volume and a low dose to surrounding normal tissue. The treatment strategy is based on intersecting multiple beams onto the target volume. Treatment plans that have the radiation dose distribution shaped to fit the target volume are known as conformal plans. Several computer-based approaches to finding highly conformal treatment plans are now being studied. The most important include:

1) Using a sophisticated search (for example, simulated annealing) to find the treatment plan that best satisfies some prespecified constraint. These specifications may be very simple, such as "find all plans that deliver 7000 cGy to the nasopharynx but no more than 5000 cGy to the temporal lobes" or very sophisticated, such as demanding that the combined probability for tumor control and lack of normal tissue complication be maximized. Some progress along these lines has already been achieved. ${ }^{1}$

2) Specifying the desired dose distribution in advance, and using algebraic methods to calculate the treatment plan that best matches this distribution. These methods fall under the general category of the "inverse problem" and progress has been made with this approach as well. ${ }^{2}$

Other approaches (less well studied) include:

3) Establishing a data base of previously designed successful treatment plans and using rule-based computer systems to determine which treatment plan template best matches the current need. These artificial intelligence approaches are only now beginning development. ${ }^{3}$

4) Searching for radiation beams that either minimize the volume of normal tissue through which they pass, or that avoid a specified structure altogether. ${ }^{4,5}$

5) Miscellaneous algebraic methods. $^{6}$

Although these approaches are interesting, and all have strong supporters, they all have serious difficulties that must be overcome before they can be clinically useful. Among these problems are the sheer size of the search space (an astronomical number of treatment plans may be possible for any given tumor), and the lack of knowledge of how to parameterize such diverse 
factors as tumor control probability, normal tissue complication probability, the patient's psychological state (willingness to sustain a complication), institutional practices, and even the prevailing malpractice climate so as to search for a "best" treatment plan. $^{7}$ In addition, completely automated optimization techniques remove a degree of understanding and control from the responsible clinician.

\subsection{Motivation and history of our project}

At the University of North Carolina we have long felt that methods that involved the physician more closely in the treatment planning process, and that relied less on biologic models would be preferable to these approaches. Specifically, we believed that a system that could calculate and display three-dimensional radiation dose distributions for arbitrary beam arrangements as fast as they could be specified would permit the physician user to exhaustively study and modify a given treatment plan, whether it was human or algorithmically conceived. With time, a skilled user could even develop a better understanding of how treatment beam changes affect the dose distribution. However, until recently, the development of a system that would allow near real-time radiation treatment planning seemed to be an impossible task.

In 1989 the Departments of Radiation Oncology (UNC-RO) and Computer Science (UNC-CS) at the University of North Carolina (UNC) formed a consortium with BellSouth Corporation, GTE, and the Microelectronics Center of North Carolina (MCNC) to prepare a response to a high speed network initiative proposed by the National Science Foundation (NSF), and the Defense Advanced Research Projects Agency (DARPA) and coordinated through the Corporation for National Research Initiatives (CNRI). The initiative was aimed at developing the technology required to boost computer networking speeds to the gigabit per second (1 billion bits/second) level. Our consortium, known as VISTAnet, proposed to use interactive radiation therapy treatment planning as the principal driving problem that would require near-gigabit networking. In the fall of 1990 it was announced that five test bed sites would be funded, including VISTAnet. ${ }^{8}$ The VISTAnet project is now at the end of its fourth year; the clinical and technical aspects of the project have been discussed in the literature, ${ }^{9}, 10$ but little attention has been paid to the difficult interactive visualization problems that had to be tackled throughout its course.

\section{THE VISTANET INTERACTIVE 3D RTP SYSTEM}

\subsection{Components}

Currently, our test bed ${ }^{11}$ features a dedicated near-gigabit/second communications network connecting the following three sites (Figure 1):

1) UNC-RO, located on the Chapel Hill campus, with a high-end graphics workstation, termed Medical Workstation (MWS).

2) MCNC, located approximately 18 miles from the UNC campus, with a 4-processor Cray Y-MP supercomputer.

3) UNC-CS, also on the Chapel Hill campus, with Pixel-Planes 5, an experimental high-performance graphics engine designed and built in UNC-CS.

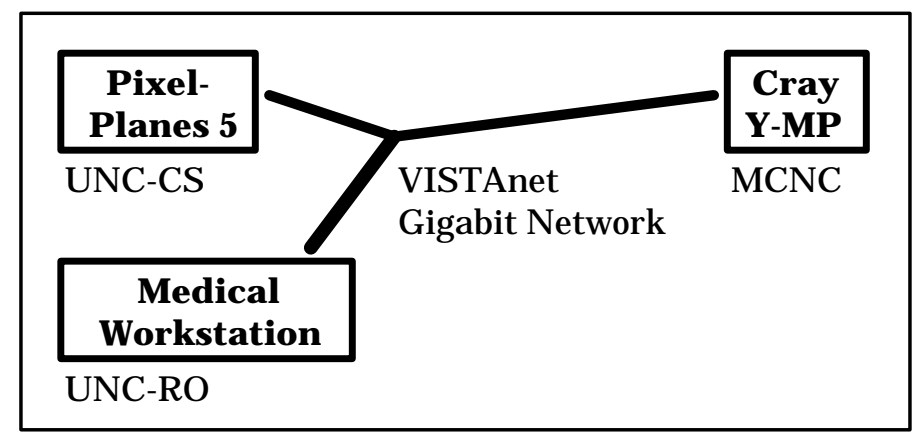

Figure 1: VISTAnet test bed sites. 
VISTAnet researchers have developed an interactive, distributed, parallel software system executing on the three computational resources enumerated above. It allows a physician user to interactively steer a supercomputer simulation of radiation treatment and thus develop and modify radiation treatment plans, using a real-time visualization for feedback. The overall task distribution among the three resources is as follows:

1) MWS: Overall system control, user interface. This subsystem controls the data flow across the high-speed network and issues commands to the other 2 components. The user interface provides the user with numerous buttons, sliders and dials that control the simulation, as well as a number of wireframe representations of the patient anatomy and treatment setup.

2) Cray: High-speed radiation dose computation. This subsystem is essentially a highly optimized treatment parameter to radiation dose processor for a specific patient anatomy.

3) Pixel-Planes 5: Interactive 3D Visualization. This subsystem provides the user with visual feedback about how the current treatment scenario affects the anatomy.

The network communication infrastructure is another essential component of the system and has been described in the specialized literature. ${ }^{11}$

\subsection{Operation}

The physician user works at the MWS (Figure 2). The MWS software allows the user to load anatomy data sets (we use presegmented volumetric CT data) for a particular study and define or modify radiation treatment parameters (such as position, orientation, shaping, and wedging) for each of a variable number of treatment beams. The parameters are transmitted over the gigabit network to the Cray, which computes the dose intensity produced within the anatomy by the specified treatment beam configuration. The (volumetric) dose data is transferred over the high-speed network to Pixel-Planes 5, which produces a combined 3D image of anatomy, treatment parameters, and resulting dose; this image is displayed at the physician user's workstation, who examines the visualization and continues to adjust the treatment parameters. The latter action prompts the entire communication-computation process to be repeated. The current processing rate is several such adjustments per second for anatomy data sets containing about a million voxels (by comparison, a physician preparing a treatment plan using conventional methods has to wait minutes to hours to be able to examine the radiation dose distributions resulting from a single tentative scenario).

\section{INTERACTIVE 3D VOLUME VISUALIZATION}

Creating and improving a 3D treatment plan is a complex task requiring thorough understanding of shape and radiation sensitivity of the anatomical structures involved. It places high demands on real-time visualization of the results: The tentative treatment scenario can change several times per second; the visualization subsystem executing on Pixel-Planes 5 must hence be able to quickly convey the scenario's characteristics to the user. In other words, it must help the user quickly comprehend spatial relationships among simultaneously displayed, geometrically complex dose and anatomy structures.

\subsection{Visualization requirements}

The answers to questions such as

-What is the radiation dose at some specific point in the anatomy?

- Which parts of the anatomy receive a specific radiation dose?

must be immediately obvious from the visualization presented to the physician user; hence the graphic content of the display must include

1) the (static) anatomy data as context for

2) the (dynamically changing) radiation dose distribution.

Two additional requirements emerged over the course of the project: 
3) The capability to label areas of the anatomy data set as belonging to specific organs or to tumor volumes, required in order to ease orientation and spatial comprehension, to avoid damage to vital and/or particularly sensitive healthy organs (for example, spinal cord or kidney), and to improve targeting on the cancerous tissue.

4) The capability to display significant information about the radiation beam configuration, thought to ease comprehension of the way treatment beam changes affect the dose distribution.

Several major problems had to be overcome to satisfy these requirements. First, interactive frame rates had to be achieved. Second, 3D display of multiple dose levels was needed; this is a particularly difficult task because simply adopting different colors for different dose levels can be confusing in 3D where one has to view one dose level through another. Finally, the visualization had to combine the various display elements in a meaningful way for the viewer.

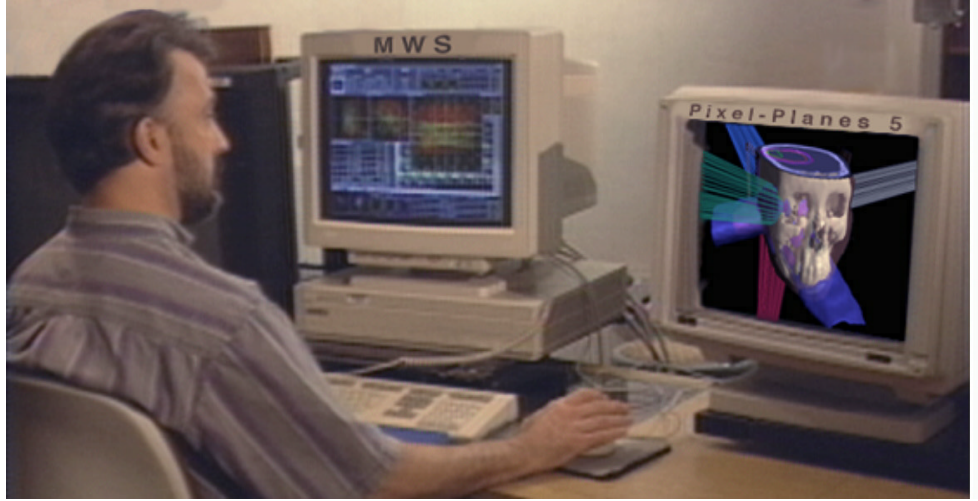

Figure 2: Medical workstation user operating the interactive radiation treatment planning system (Pixel-Planes 5 image simulated).

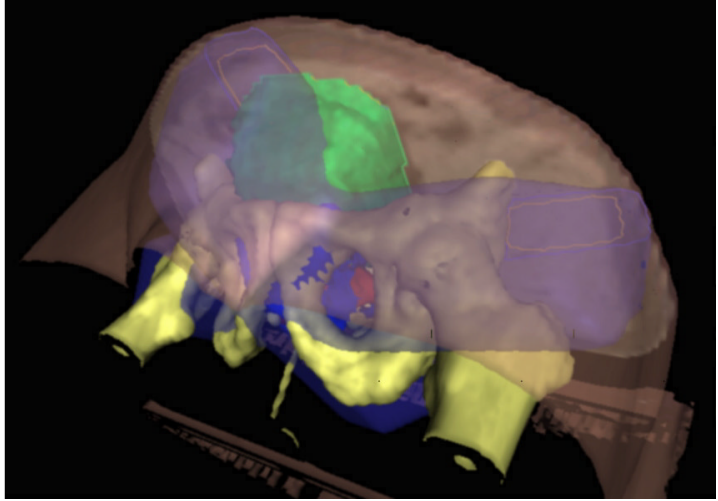

Figure 3: Visualization showing the pelvis of a prostate cancer patient. Anatomy is represented via a translucent skin isosurface (brown), an opaque bone isosurface (yellow), and pre-segmented anatomical structures: prostate (red), bladder (green). A cutaway plane shows radiation dose isocurves. The translucent and opaque blue elements are dose isosurfaces.

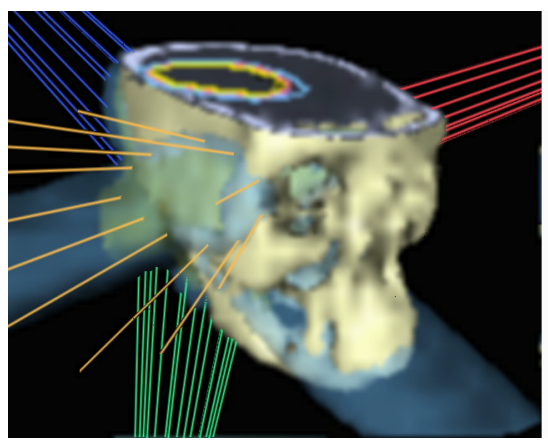

(a)

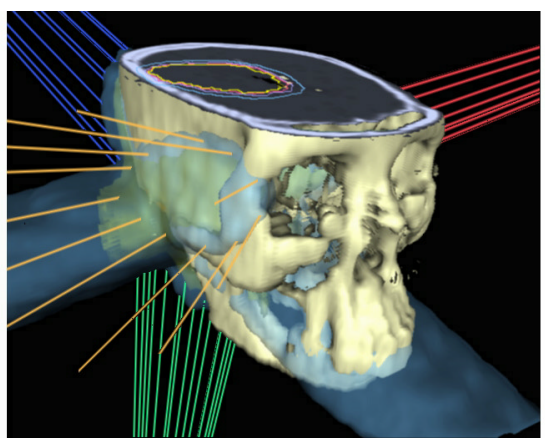

(b)

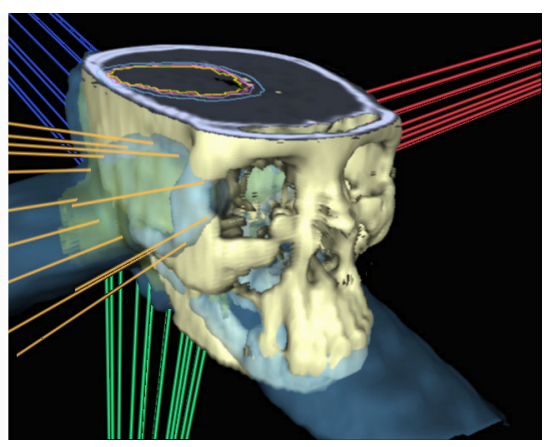

(c)

Figure 4: (a) Low-resolution image, rendered in approximately 0.1 sec on Pixel-Planes 5. (b) High-resolution image, rendered in approximately 3 sec. (c) Stereo complement to image in (b). The wireframes represent treatment beams. 
The current features and capabilities of the visualization are the result of repeated interaction between physician users and graphics software designers, as well as much trial-and-error; nowadays, a typical Pixel-Planes 5 VISTAnet visualization image is a mixture of volume renderings, surface drawings, and line drawings; it consists of

- a volume rendering of anatomy merged with

- a volume rendering of dose isosurfaces, combined with

- a cutaway plane showing original volume data, along with

- radiation dose isocurves and

- radiation beam outlines presented as wireframes.

Figure 3 shows such an image. Both the static and the dynamic data sets are represented by multiple, user-changeable, superimposed, interpenetrating, translucent or opaque surfaces. For the anatomy, the surfaces are usually set to delineate tissue boundaries such as skin or bone surfaces, or previously identified structures such as internal organs or tumor volumes. For the dose, the isosurfaces show multiple radiation dose threshold levels. The cutting plane with its radiation dose isocurves (familiar from conventional 2D treatment plans) is often moved quickly by the user to different places in the patient anatomy to gather detailed information about local dose levels. The stereo pair in Figures $4 \mathrm{~b}$ (left) and $4 \mathrm{c}$ (right) shows a treatment scenario for which shape, position, and orientation of the treatment beams are indicated by wireframe elements.

\subsection{Implementation}

The visualization system, developed jointly by UNC-RO and UNC-CS researchers, uses at its basic algorithmic level some of the principles outlined by volume rendering researchers. ${ }^{12,13,14,15,16}$ The initial experimental renderer was also briefly described. ${ }^{17}$ The mapping of the volume rendering algorithms used in VISTAnet onto the heterogeneous Pixel-Planes 5 architecture is extremely complex; ${ }^{18}$ the brief overview given here emphasizes the specifics of interactive visualization for VISTAnet's 3D RTP application.

The hardware platform for the visualization subsystem is Pixel-Planes 5, a high-performance multiple-instruction-multiple-data (MIMD) graphics engine with general-purpose computing nodes based on the Intel i860 ${ }^{\mathrm{TM}}$ microprocessor, and special-purpose rendering nodes based on massively parallel single-instruction-multiple-data (SIMD) processor-enhanced memories. ${ }^{19}$ All nodes are interconnected via the system's internal 5 Gigabit/sec token ring network. Also connected to the token ring are frame buffers, the Network Interface Unit (NIU) providing access to the gigabit network (which is external to Pixel-Planes 5), and the Sun-4 host computer.

The visualization software uses a data- and image-parallel ray casting algorithm mapped onto the Pixel-Planes 5 architecture (Figure 5). The image generation pipeline consists of sampling nodes and compositing nodes; the sampling nodes traverse the anatomy and the dose volume data sets and transmit image fragments to the four compositing nodes, where they are assembled into the final image. Most of the machine's general-purpose computing nodes are allocated to the sampling task, the most compute-intensive sub-task of the visualization system. To obtain satisfactory frame rates, the (static) anatomy voxels are stored with precomputed normals and threshold bits to speed up ray processing. A total of 40 bits are used for a static voxel, including flag bits for anatomical structures; these are predefined by hand contouring on each slice of the anatomy data set and scan converted into it at system startup time.

The (dynamic) dose data set from the Cray ( 8 bits per voxel) is transmitted into the graphics engine through the NIU. Multiple parallel daisy-chains (Figure 6) are used to replicate the incoming radiation dose on all sampling nodes, where it must be available for image fragment generation. Dose reception and response to other visualization commands (such as translating or rotating the view) are asynchronous, with priority given to the dose reception; i. e., new incoming dose from the Cray will cause ongoing computation of the next frame to be preempted and restarted after the dose array has been updated. Visualization commands from the MWS are also received through the NIU; in addition to modifying the view and the lighting direction, the user can change colors and translucency levels for each isosurface or wireframe element in the display. The thresholds for each anatomy and radiation dose isosurface and cutplane isocurve are also user-modifiable.

The Pixel-Planes 5 based visualization subsystem can be operated within VISTAnet (under MWS control) or as a standalone general-purpose volume visualization system. It has its own user interface, which contains a superset of the visualization controls accessible through the MWS user interface. This not only provides access to all of the program's functions, but also allows independent experimentation and development/debugging of new visualization features. 
static/dynamic

volume data sets,

partitioned into

slabs, each assigned

to groups of

sampling nodes

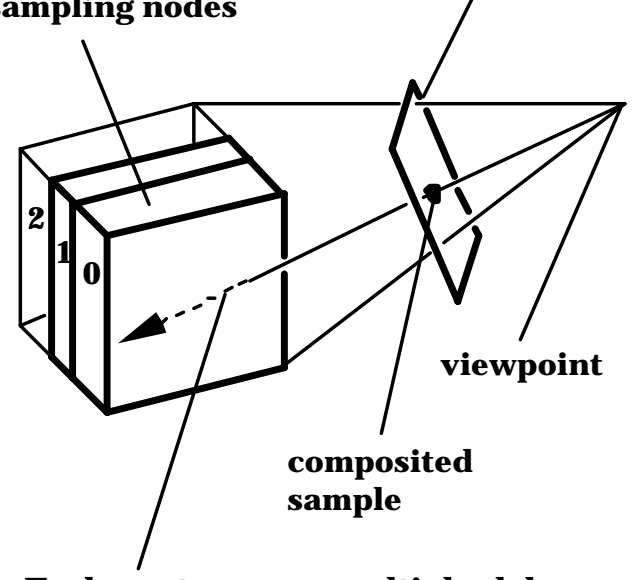

Each ray traverses multiple slabs;

each image fragment sample

calculated by a sampling node from

a different group

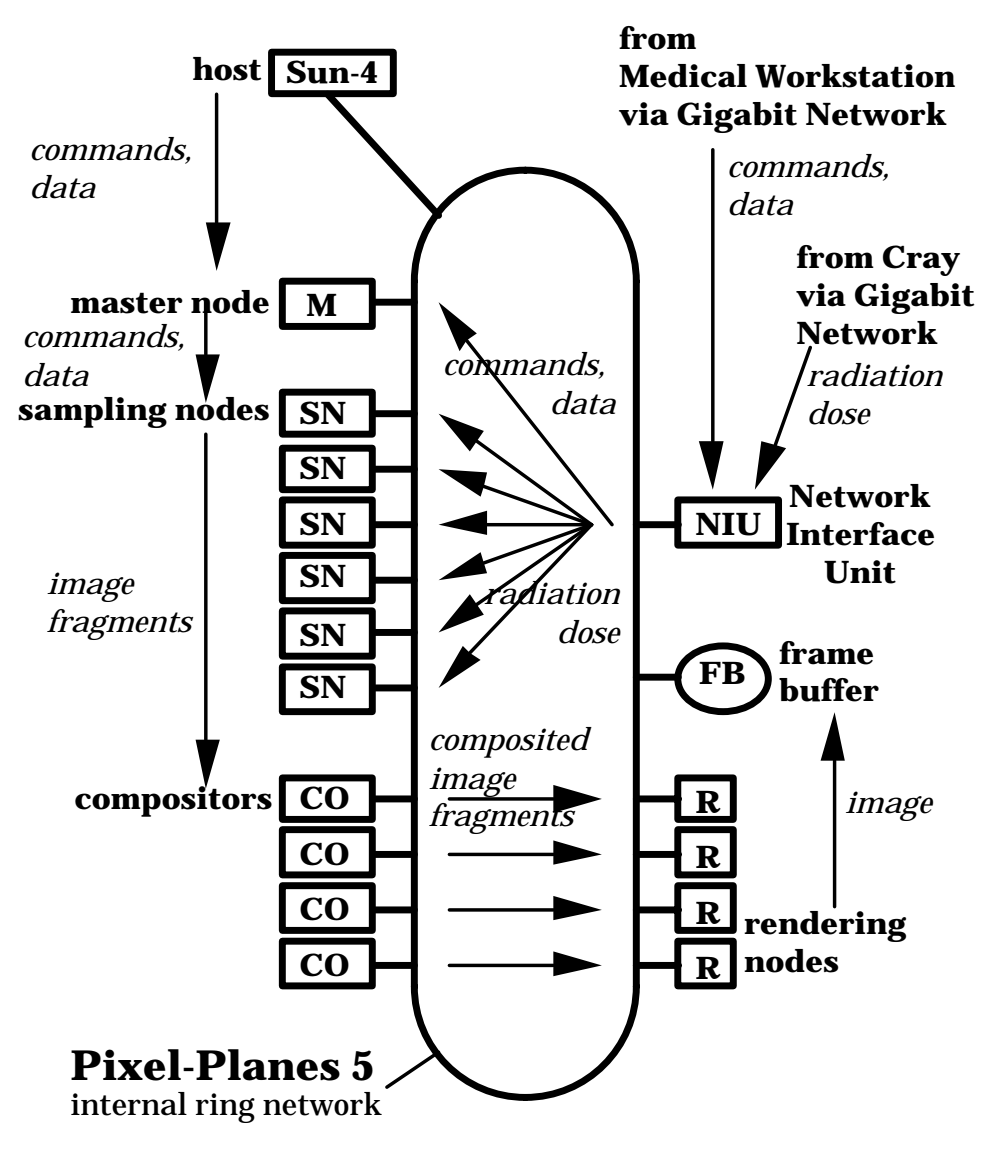

Figure 5: Visualization algorithm (left); Pixel-Planes 5 architecture and visualization algorithm data flow (right).

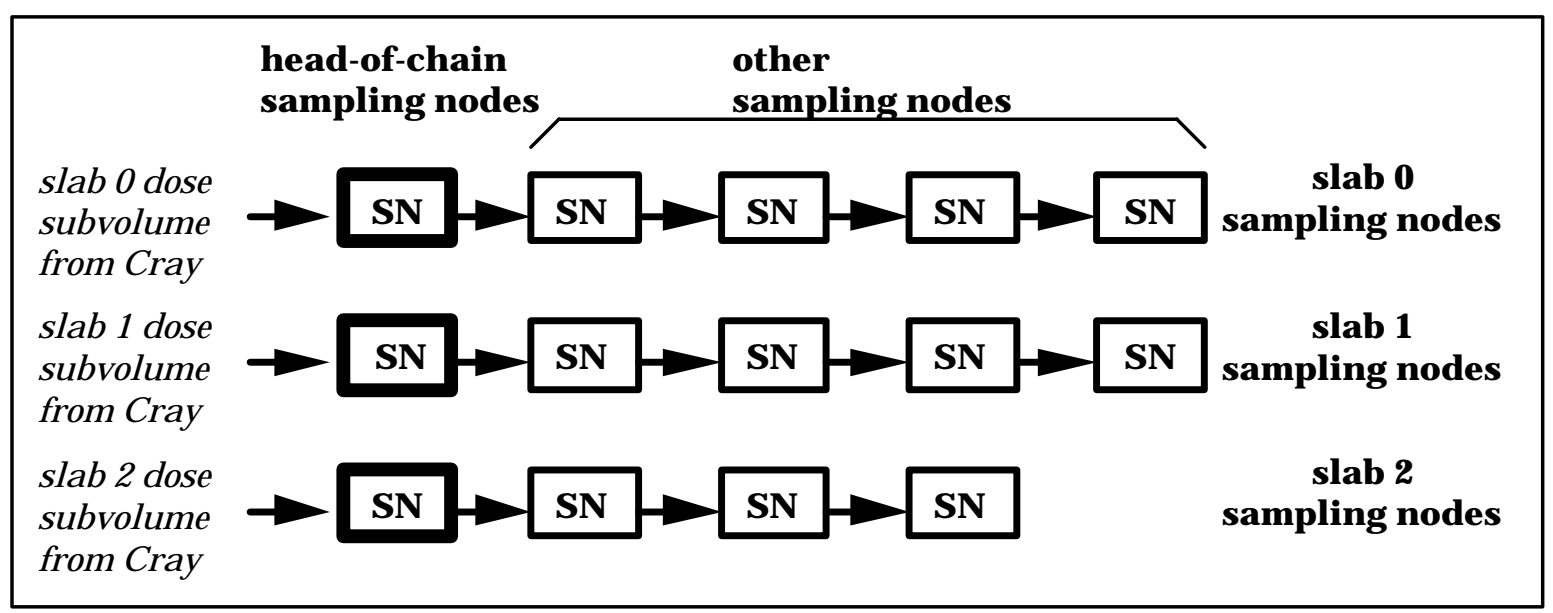

Figure 6: Multiple daisy-chain transmission and distribution of dose data to the sampling nodes. 


\subsection{Depth cues, performance, and image quality}

It takes several seconds to generate a high-resolution VISTAnet image on Pixel-Planes 5; we therefore had to judiciously arbitrate the allocation of graphics computing resources between image quality and image generation speed when designing the visualization system; without sufficient quality the user cannot see necessary details in the image; without sufficient speed, the user cannot fully comprehend spatial relationships due to lack of depth cues (such as the kinetic depth effect), which diminish rapidly with decreasing frame rate.

We adopted a resolution/speed tradeoff, sacrificing some image space resolution in favor of higher frame rates, in particular in image areas which are fairly uniform in appearance (adaptive sampling). Furthermore, the system displays coarse frames at interactive rates and takes advantage of user pauses to produce a gradually refining image (progressive refinement) (Figure 4).

The integrated adaptive-sampling-progressive-refinement operation is made flexible by giving the user full interactive control over the resolution/speed trade-off parameters. Kinetic depth effect for high-quality images is provided by appending to the progressive refinement sequence a series of 7 highest-resolution frames displaying the visualized structures in animated oscillatory rotation (rocking cineloop). Thus, if the user pauses long enough, he/she will notice gradually increasing image resolution, followed by increasingly smooth left-right rocking of the displayed high-resolution structures.

Finally, standard depth cues such as directional lighting (currently from a single parallel light source) with diffuse and specular illumination are also used.

\subsection{RTP under VISTAnet}

As explained above, advances in VISTAnet visualization are first implemented in experimental versions of the standalone renderer. As new features and visualization improvements become available, they are made available to the VISTAnet system; currently, all of the features described above are accessible to the MWS user, but research on new visualization features is ongoing.

The modus operandi for UNC-RO researchers when defining and modifying a treatment plan is to begin by simultaneously examining several isosurfaces within the patient anatomy. For example, when working on a prostate radiation treatment plan one might wish to see the skin and bones as anatomic landmarks. However, by simply adjusting one of the anatomy isosurface levels, muscles and bones might be seen. The prostate itself cannot be volume rendered directly from the data set as its surface is too ill defined to be segmented in this way. It can be displayed as a separate anatomical isosurface if it has been predefined and scan converted into the data set as explained above. The treatment plan itself and its effects are examined via the wireframe beam representations and the 3D radiation dose isosurfaces, each of which is attached to a radiation dose isocurve on the movable cutting plane. In practice one usually starts with several elements displayed and eventually eliminates everything but the tumor volume and radiation dose display as the treatment parameters are fine-tuned.

The VISTAnet system allows the oncologist researcher who uses it to examine a large number of radiation dose distributions in a very short time. This system then becomes an ideal exploration vehicle for the large parameter space of treatment planning possibilities. This "exploratory" approach is fundamentally different from the computer-based RTP methods generally studied at other institutions, such as the already mentioned simulated annealing and inverse methods. Both of these approaches have the drawback that they require the treatment planner to know what dose distribution and/or tissue damage they are willing to accept in advance. However, in our opinion, what the clinician really wants is the "best possible plan for a given case." It is often not possible to state in advance what that plan is, but the clinician "knows it when he sees it." This is one of the motivations for the approach taken by VISTAnet.

Using VISTAnet's interactive dose calculation and display capabilities, a number of new treatment plans have emerged. It is gradually becoming clear that certain geometric rules are important in setting up optimized plans which are superior to those resulting from conventional 2D slice-based methods. For example, radiation beams should overlap spatially as little as possible. This rule has led to the design of new treatment plans which make full use of non-coplanar beams, such as the tetrahedral beam arrangement ${ }^{20}$ that may be near optimal for situations where there is spherical symmetry, such as in the brain. Another approach is the six-field hexad, which is cylindrically shaped and may be near ideal for the prostate. Research into these types of treatment plan optimizations remains ongoing. 


\section{CONCLUSIONS AND FUTURE WORK}

VISTAnet, an interactive dose calculation and display system running on a metacomputer via a gigabit network, can calculate and display an entire 3D radiation dose distribution in less than one second. As such, the system can be used to explore many treatment planning possibilities that could not be studied on a lesser system. Thus, the ability to rapidly calculate and display radiation dose distributions can make a major contribution to the understanding of radiation dose optimization.

The 3D treatment plan definition/optimization task in VISTAnet is akin to interactively steering the radiation dose supercomputation based on real-time visualization of the treatment scenario-hence the importance of high-quality real-time imagery. However, it has been extremely difficult to achieve the current visualization frame rates despite the availability of a highest-performance graphics engine. To do so, we had to specifically target and optimize much of the algorithms to Pixel-Planes 5. We are not satisfied with the current performance/resolution tradeoff since it weakens the kinetic depth cues. We have made both high frame rates and kinetic depth effect available in our system; however, lack of sufficient computing power has so far prevented us from offering high frame rates together with high-resolution images, in other words: interactive kinetic depth. There is therefore a need for significantly increased image generation speed.

On the next-generation UNC graphics engine $\left(\right.$ PixelFlow $\left.^{21}\right)$ a VISTAnet-like visualization could benefit from at least a factor of 5 speedup, and considerably more by taking advantage of that system's image-based texturing capability. In fact, such texturing capabilities are available today on high-end graphics workstations such as the Silicon Graphics Reality Engine, if we accept limitations such as lower-speed radiation dose transmission into the graphics engine and fixed lighting. As Figure 4 demonstrates, VISTAnet-type visualizations could easily accommodate speedups of 2 orders of magnitude.

As high-bandwidth networked systems such as VISTAnet become more ubiquitous, we expect visualization systems that are embedded within tightly coupled supercomputing networking environments to become more commonplace. We hope that our experimental work in this area will contribute towards the usefulness and effectiveness of such systems.

\section{ACKNOWLEDGMENTS}

We thank Stephen M. Pizer (UNC-CS Senior Investigator) and Scott Sailer, M. D. (UNC-RO physician user) for their significant contributions and constructive criticism. The following former and current UNC-CS graduate research assistants have contributed algorithms and/or software: Suresh Balu, David T. Chen, Hong Chen, Qin Fang, Matt Lavoie, Jonathan McAllister, Ulrich Neumann and John Rhoades.

This work was supported by NSF and ARPA under Cooperative Agreement NCR-8919038 with CNRI ("VISTAnet: A Very High Bandwidth Prototype Network for Interactive 3D Imaging"), by BellSouth, and by GTE.

\section{REFERENCES}

Niemierko, A., M. Urie, M. Goitein, "Optimization of 3D radiation therapy with both physical and biological end points and constraints," Int. J. Radiat. Oncol. Biol. Phys., Vol. 23, pp. 99-108, 1992.

2 Källman, P., B. K. Lind, A. Brahme, "An algorithm for maximizing the probability of complication free tumor control in radiation therapy," Phys. Med. Biol., Vol. 37, pp. 871-890, 1992.

3 Kalet, I. J., J. P. Jacky, "Knowledge-based computer simulation for radiation therapy planning," Proceedings of the 9th International Conference on the Use of Computers in Radiation Therapy, edited by A. D. Bruinvis, P. H. van der Giessen, H. J. van Kleffens, and F. W. Wittkämper. Amsterdam, Elsevier Science Publishers B.V., 1987, pp. 553-556. (Also, personal communication.)

4 Chen, G. T. Y., D. R. Spelbring, C. A. Pelizzari, J. M. Balter, L. C. Myrianthopoulous, S. Vijayakumar, H. Halpern, "The use of beam's eye view volumetrics in the selection of non-coplanar ports," Int J. Radiat. Oncol. Biol. Phys., Vol. 23, pp. 153-163, 1992.

5 McShan, D.: Personal communication [manuscript to be published]. 
Rosen, I. I., R. G. Lane, S. M. Morrill, J. A. Belli, "Treatment plan optimization using linear programming," Med. Phys., Vol 18, pp. 141-152, 1991.

Jain, N. L., M. G. Kahn, R. E. Drzymala, B. Emami, J. A. Purdy, "Objective evaluation of 3-D radiation treatment plans: A decision-analytic tool incorporating treatment preferences of radiation oncologists," Int J. Radiat. Oncol. Biol. Phys. [In press].

Stix, G., "Gigabit connection," Scientific American 263, pp. 118-120, October 1990.

Rosenman, Julian, Tim J. Cullip, "High-performance computing in radiation cancer treatment, Crit .Rev. Biomed. Eng., Vol. 20, pp. 391-402, 1992.

Rosenman, Julian, E. L. Chaney, Tim J. Cullip, J R. Symon, Henry Fuchs, D. Stevenson, "VISTAnet: Interactive Real-Time Calculation and Display of 3-Dimensional Radiation Dose: An Application of Gigabit Networking," Int. J. Radiat. Oncol. Biol. Phys., Vol. 25, pp. 123-129.

Stevenson, Daniel, Julian Rosenman, "VISTAnet Gigabit Testbed. IEEE Journal of Selected Applications of Communications, Vol. 10, pp. 1413-1420, 1992.

Sabella, Paolo, "A Rendering Algorithm for Visualizing 3D Scalar Fields," Computer Graphics, Vol. 22(4) (Proceedings of SIGGRAPH '88), pp. 51-58, July 1988.

14 1988

Levoy, Marc, "Design for a Real-Time High-Quality Volume Rendering Workstation," Proceedings of the Chapel Hill Workshop on Volume Visualization, pp. 85-92, 1989.

Westover, Lee, "Interactive Volume Rendering," Proceedings of the Chapel Hill Workshop on Volume Visualization, 1989, pp. 9-16, 1989.

17

Yoo, Terry S., Ulrich Neumann, Henry Fuchs, Stephen M. Pizer, Tim Cullip, John Rhoades, and Ross Whitaker, "Direct Visualization of Volume Data," IEEE Computer Graphics and Applications, Vol 12, Number 4, pp. 63-71, July 1992.

Neumann, Ulrich, Andrei State, Hong Chen, Henry Fuchs, Tim J. Cullip, Qin Fang, Matt Lavoie, John Rhoades, "Interactive Multimodal Volume Visualization for a Distributed Radiation-Treatment Planning Simulator, " UNC-CS Technical Report TR94-040, June 1994.

19 Fuchs, Henry, John Poulton, John Eyles, Trey Greer, Jack Goldfeather, David Ellsworth, Steve Molnar, Greg Turk, Brice Tebbs, Laura Israel, "Pixel-Planes 5: A Heterogeneous Multiprocessor Graphics System Using Processor-Enhanced Memories," Computer Graphics, Vol. 23(3) (Proceedings of SIGGRAPH '89), pp. 79-88, July 1989. separation as a starting point for noncoplanar 3-D treatment planning," Int. J. Radiat. Oncol. Biol. Phys., [in press], 1994.

21 Molnar, Steven, John Eyles, John Poulton, "PixelFlow: High-Speed Rendering Using Image Composition," Computer Graphics, Vol. 26(2) (Proceedings of SIGGRAPH '92), pp. 231-240, July 1992. 